\title{
Responses by four Local Research Ethics Committees to submitted proposals
}

\author{
Gerry Kent University of Sheffield, Sheffield
}

\begin{abstract}
Background-There is relatively little research concerning the processes whereby Local Research Ethics Committees discharge their responsibilities towards society, potential participants and investigators.
\end{abstract}

Objectives - To examine the criteria used by LRECs in arriving at their decisions concerning approval of research protocols through an analysis of letters sent to investigators.

Design-Four LRECs each provided copies of 50 letters sent to investigators after their submitted proposals had been considered by the committees. These letters were subjected to a content analysis, in which specific comments and requests for additional information and changes in the protocols were recorded and compared.

Findings-Overall $24 \%$ of proposals were approved without request for changes or clarifications, but this varied by committee: one committee approved only $6 \%$ of proposals without change or clarification while the others ranged from $26 \%$ to $32 \%$. The content analyses of responses indicated that they could be placed into four categories: (i) further information for the committee to aid in their deliberations; (ii) requests for changes to the design or justification for the design used; (iii) changes to the information sheets provided to potential participants; and (iv) changes to consent procedures. Of these, alterations to information sheets were the most common type of request. These four types of response could be seen as safeguarding the wellbeing of potential participants (the principle of non-maleficence), of promoting the scientific validity of the research (the principle of beneficence), and of enhancing the rights of potential participants (the principle of autonomy).

Conclusions-The committees were consistent in the types of requests they made of investigators, which can be seen as attempts to protect participants' rights and ensure the scientific validity of studies. Without an analysis of the proposals sent to the committees, however, it is difficult to account for the variation in the requirements set by the committees before approval was given.

(Fournal of Medical Ethics 1999;25:274-277)

Keywords: Ethical review
There has been much interest recently in the practice of Local Research Ethics Committe

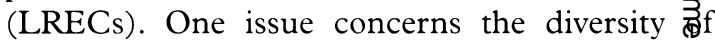
LREC requirements before giving approve Gilbert et $a l^{1}$ and Harries et al pointed out thicat there is considerable variation between decisions made by different committees, resulting in mus disquiet within the research community. ${ }^{3}$ Foster argues that this may be due to different committees holding different values, with one committofe perhaps valuing the consequences of research, another the rights of potential participan 8 . Garfield ${ }^{4}$ reported that there was a considerable range in time required for approval (2.2 to 54 weeks, mean 9 weeks) and the number of changess requested (range from 0 to 4 , median 1.5) for the 13 ethics committees that were approached approval for a postal survey.

Another issue concerns the criteria used LRECs in arriving at their decisions. Several attempts have been made to ascertain these critria. In Garfield's study, most comments from committees to investigators involved the proce्तs of obtaining consent, but there were also concer about confidentiality. Harding and $\mathrm{Ummel}^{\top}$ exarmined the issues in more depth by reviewing eight protocols which had previously been given LRE€ approval. As judged by a simulated review panel, only three protocols were considered to provite fully adequate information to allow an ethicall review, while for five other proposals addition safeguards were considered necessary, particulany covering informed consent. One method whis would give more detail about the process of ethe cal review would be to analyse recordings of the actual discussions which take place duriteg committee meetings. However, it seems likely th recording would itself affect the process. An alternative would be to analyse the letters sent investigators, exploring the themes cited ard requests for changes made. This approac although it relies on a distillation of the discusions, poses fewer issues of privacy and confidentiality (the individuals making points and the investigators would not be identified), and if do retrospectively would not alter the review proces. 
The aims of the present study were (a) to examine the content of letters sent to investigators by LRECs as a result of their deliberations and (b) to examine any differences between committees in terms of the number and type of comments made.

\section{Methods}

After consultation with the author's own LREC, letters were sent to the chairs of five LRECs in the Trent Region, UK, describing the nature and purpose of the study. The letter requested copies of the responses to the first 50 full submissions beginning January, 1996. Confidentiality of both the committee and the investigators was assured. All five committees agreed to this request, subject to assurances about confidentiality and anonymity. In four instances the letters were supplied by the committees' secretaries, while in the fifth instance the author was asked to undertake the photocopying himself at the LREC premises.

After copying, each letter was subjected to a content analysis, in which "units of sense" identified. This analysis indicated that the responses could be coded into five categories: (i) requests for further information for the committee, (ii) comments on the design of the study, (iii) requests for alterations to the information sheet, (iv) requests for alterations to the consent form, and (v) no alterations requested. Numerical data were collected in two ways. First, each letter was coded according to whether each category of comment was or was not present in the letter. Second, the number of comments within each category was noted. For example, an issue of the validity of a study might be mentioned more than once - it could involve both the process of randomisation and the use of unblinded assessments. If so, it was coded as two requests for alterations.

\section{Results}

The information provided by one LREC was unsuitable, in that the copies of responses sent included a wide variety of correspondence. This sample was therefore deleted, leaving a sample of 50 letters from each of the four remaining LRECs. In the summaries of results given below, the LRECs indicated by B, C and D were affiliated with a teaching hospital, while A was not.

\section{Content analysis: types of request for changes in protocols}

The comments made by the committees could be placed into four categories: requests for additional information for use by the LREC; comments on the design of the study, which involved questions about its validity; requests for changes in the information sheet, and requests for changes in the consent procedures.

REQUESTS FOR ADDITIONAL INFORMATION FOR USE BY THE LREC

Comments were placed in this category when the letter indicated the committee could not make a decision based on the information provided by the investigator(s). These issues concerned the background to the study. Although such issues might not be apparent to participants, nevertheless they could affect them in some way. Queries included clarification about the involvement of other clinical and non-clinical staff (such as any risks they might encounter), insurance issues, the provision of supervision for researchers collecting data, Clinical Trial Certificate Exemption (CTX) and other certificates, and financial support. Perhaps most centrally, LREC members requested assurances that the participants would not be placed at unacceptably high risk. This could occur with a new form of treatment, with withdrawal of treatment during a period of time for the purposes of the research, or with the dangers involved in extra investigations. Thus, this category of comments could be considered as being primarily concerned with the avoidance of harm. ${ }^{9}$

\section{COMMENTS ON THE DESIGN OF THE STUDY}

There has been some discussion concerning whether it is appropriate for LRECs to comment upon, and to take the scientific validity of, a study into account when considering ethical approval. This is an important area of debate, since members of the committee may not be familiar with all the types of design which are presented to them. For example, relatively few members may be familiar with qualitative designs which, although equally demanding as quantitative designs, are used relatively rarely. Nevertheless, there is a growing acceptance that design issues are relevant to ethical review, ${ }^{10}$ and this was reflected in the comments made to researchers. Letters included requests for: more details about methods and recruitment procedures; a power analysis, and an indication of how the results would be analysed. Letters also included queries about the feasibility of a study, given either the financial resources or the time available to the investigator, and whether a study was necessary, given previous research in the area. Issues of validity included queries about methods of randomisation, the selection of control groups, the validity and reliability of particular assessments and the need for "blinded" assessments. These types of issues thus involved attempts to ensure that the research 
would have value to society and the scientific community and so could be seen as being concerned with the achievement of the greatest possible good - the principle of beneficence.

\section{REQUESTS FOR CHANGES IN THE INFORMATION SHEET}

All LRECs required that potential participants be given written information which they could use when deciding whether to take part in the research. Requests for changes in these information sheets primarily included clarification/ simplification in the explanation of the study; further details of what would be involved (particularly details concerning risks); notice that participation was voluntary; financial considerations (particularly deletions of any inducements to take part); the provision of sheets for children and others involved in the study besides the participants themselves, and compensation arrangements and complaints procedures. Thus, this category of comments was mainly concerned with informing participants of what would be involved and their rights - issues of autonomy.

\section{REQUESTS FOR CHANGES IN CONSENT PROCEDURES} This category also concerned the principle of autonomy and again reflects the central purpose of LRECs - to protect the rights of participants. Although it was rare that a consent form was not included in the submission, letters included requests for additions to the form, including an explicit indication that participants realised that their participation was voluntary, that they could withdraw at any time without having to give a reason, and that consent forms would be completed by children as well as their parents or guardians. There were also a small number of miscellaneous requests, such as that the form be printed on headed notepaper, that space for a witness's signature be provided, and for assurance that there would be adequate time between explanation of the study and the request for consent, for the participant to consider his or her decision.

\section{The incidence of categories of request by committee}

As noted above, the comments were coded in two ways: first, whether or not a category of comment was present in the letter; and second, for the number of independent comments within each category. Table 1 summarises the results for the presence of categories, by committee. The percentage (rather than absolute numbers) of letters which contained each category is illustrated. In general, it was more common for committees to
Table 1 Percentages of letters which contained the four categories of comments, by committee

\begin{tabular}{|c|c|c|c|c|c|}
\hline Committee & $\begin{array}{l}\text { Information } \\
\text { for } \\
\text { committee }\end{array}$ & $\begin{array}{l}\text { Design } \\
\text { issues }\end{array}$ & $\begin{array}{l}\text { Information } \\
\text { sheets }\end{array}$ & Consent & $\begin{array}{l}\text { No } \overline{\bar{ज}} \\
\text { changes } \\
\text { requested }\end{array}$ \\
\hline A & 30 & 14 & 58 & 16 & 26 \\
\hline B & 26 & 8 & 34 & 14 & 32 \\
\hline C & 20 & 12 & 50 & 38 & 32 \\
\hline $\mathrm{D}$ & 56 & 54 & 84 & 14 & 6 \\
\hline Mean & 33 & 22 & 57 & 21 & 24 \\
\hline
\end{tabular}

request changes than to approve a protocol wit $\overrightarrow{\mathrm{BJ}}$ out alterations of some kind: only $24 \%$ wef? approved without change. Some types of chang were more common than others. Comparisong between the columns of table 1 indicate thas changes to the information sheets formed thie most common type of requests (for $57 \%$ of att applications), followed by additional information to the committee $(33 \%)$ and then comments on the study's design and the consent form $(22 \%$ an $21 \%$ respectively): Friedman test, Chi-Square $39.8, \mathrm{df}=1, \mathrm{p}<0.001$.

However, these requests and comments wefe not spread evenly across committees. Table $\Phi$ indicates that committee $\mathrm{D}$ was more likely request additional information (Chi-Square $\overline{\bar{\sigma}}$ 17.1, $\mathrm{df}=3, \mathrm{p}<0.001$ ), to comment on the desigh of the study (Chi-Square $=40.3, \mathrm{df}=3, \mathrm{p}<0.00 \mathrm{~B}$ ) and to request changes to the information she (Chi-Square $=26.6, \mathrm{df}=3, \mathrm{p}<0.001$ ). Committe $\mathrm{C}$, on the other hand, was most likely to reque alterations to the consent form (Chi-Square 12.6, $\mathrm{df}=3, \mathrm{p}<0.01$.

Table 2 presents additional information of these changes. Here the absolute number of con ments is illustrated. The pattern is very similai: changes to aspects of the information sheet form the most common requests (Friedman $=50.4$, df $=3, \mathrm{p}<0.001$ ), and committee $\mathrm{D}$ made the most comments under the categories of information the committee (Kruskal-Wallis $=17.8, \mathrm{df}=\overline{\bar{\beta}}$ $\mathrm{p}<0.001$ ), comments on the design (KruskaP Wallis $=41.6, \mathrm{df}=3, \mathrm{p}<0.001)$ and requests $\mathrm{fo}$ changes in the information sheet (Kruskal-Walles $=38.5, \mathrm{df}=3, \mathrm{p}<0.001$ ), while Committee $\mathrm{C}$ was most concerned about consent procedures (Kruskal-Wallis $=12.3, \mathrm{df}=3, \mathrm{p}<0.01)$.

Table 2 Number of comments made by each committee for each category

\begin{tabular}{|c|c|c|c|c|}
\hline Committee & $\begin{array}{l}\text { Information } \\
\text { for committee }\end{array}$ & Design & $\begin{array}{l}\text { Information } \\
\text { sheets }\end{array}$ & Consent \\
\hline A & 20 & 8 & 40 & 10 \\
\hline B & 15 & 4 & 18 & 8 \\
\hline $\mathrm{C}$ & 12 & 7 & 42 & 21 \\
\hline D & 36 & 39 & 81 & 7 \\
\hline Total & 83 & 58 & 181 & 46 \\
\hline
\end{tabular}




\section{Discussion}

These results raise a number of issues, but it is appropriate to outline some of the methodological aspects of the current study which bear on the interpretation of the findings. First, the results have been gathered from a small number of LRECs from a restricted area. A different pattern of results might be found if a larger number of committees were surveyed from a wider geographical area. Other categories of comments or proportions of comments within categories might be uncovered. Second, the requests for further information and changes shown in the letters may not be accurate summaries of the discussions which had taken place during committee meetings. Although accuracy is likely, there is no certainty of this.

With these caveats, the results do provide additional information about the concerns and values held by the LRECs who took part in this study. There are three main conclusions. First, the LRECs took care in gathering the information they needed to make their decisions. If this information was not provided in the submitted protocols, steps were taken to acquire it. Second, the comments to investigators fell into four categories, consistent with the ethical principles of nonmaleficence, beneficence and autonomy. The principle of non-maleficence was maintained through the gathering of information from investigators which concerned risk to potential participants; the principle of beneficence was supported through a concern with the design and scientific validity of the research; and, perhaps most importantly in the eyes of the committee members, the principle of autonomy was promoted through careful assessment of both information sheets and consent procedures. Thus these results are consistent with the findings of other studies on the process of ethical approval. ${ }^{11}$

Third, there was statistical variation between the types and numbers of comments made by the committees. While committee $\mathrm{C}$ was the most concerned about consent issues, committee $\mathrm{D}$ was the most likely to request additional information from investigators, most likely to comment on design issues and most likely to request changes to information sheets. It is not clear why this was the case. There is the possibility that applications to this committee were less well developed or competently completed. However, like committees B and C, it is closely associated with a teaching hospital and most applications came from experienced researchers. Alternatively, the studies may have involved a greater number of ethically problematic issues, such as the assessment of novel treatments. This could account for the requests for additional information from investigators and the crucial importance of complete and understandable information sheets. A third possibility is that the personnel attached to committee $\mathrm{D}$ are more stringent in their use of criteria, requiring greater clarity or having higher standards for design considerations. Without access to the submitted research proposals it is difficult to assess the validity of these possibilities.

Future studies in this area could examine the process of LREC approval in different ways. Clearly, an analysis of submitted proposals would be of benefit. Although there would be ethical issues involved in such a study - particularly confidentiality - it might be possible to examine the ways in which committees do (or do not) identify relevant issues. Further, although the amount of research on LRECs is growing, we know very little about the fate of research projects which do not receive approval. Nor is much known about the processes whereby investigators decide to take a proposal to an ethics committee. This is especially relevant to audits, where the boundary between research and monitoring of care can be drawn only with difficulty.

\section{Acknowledgement}

The author wishes to thank the Sheffield Community Health Council who supported this project.

Gerry Kent, PhD, is Lecturer in Clinical Psychology, Department of Psychology, University of Sheffield, Sheffield.

\section{References}

1 Gilbert C, Fulford K, Parker C. Diversity of the practice of district ethics committees. British Medical fournal 1989;299:1437-9.

2 Harries H, Fentem P, Tuxworth W, Hoinville G. Local research ethics committees. Fournal of the Royal College of Physicians of London 1994;28:150-4.

3 Middle C, Johnson A, Petty T, Sims L, Macfarlane A. Ethics approval for a national postal survey: recent experience. British Medical fournal 1995;311:659-60.

4 Garfield P. Cross district comparison of applications to research ethics committees. British Medical fournal 1995;311:660-1.

5 While A. Ethics committees: impediments to research or guardians of ethical standards? British Medical fournal 1995;311:661.

6 Foster C. Why do research ethics committees disagree with each other? Fournal of the Royal College of Physicians of London 1995;29:315-18.

7 Harding T, Ummel M. Evaluating the work of ethical review committees: an observation and a suggestion. Fournal of Medical Ethics 1989;15:191-4

8 Dey I. Qualitative data analysis. London: Routledge, 1993.

9 Beauchamp T, Childress J. Principles of biomedical ethics [4th ed]. Oxford: Oxford University Press, 1994.

10 Rosenthal R. Science and ethics in conducting, analysing and reporting psychological research. In: Bersoff D, ed. Ethical conflicts in psychology. Washington, DC: American Psychological Association, 1995:357-63.

11 Kent G. The views of members of Local Research Ethics Committees, researchers and members of the public towards the roles and functions of LRECs. Fournal of Medical Ethics 1997;23:186-90. 